\title{
Eye-movement replay supports episodic remembering
}

Roger Johansson ( $\sim$ Roger.Johansson@psy.lu.se )

Lund University https://orcid.org/0000-0003-3434-2538

Marcus Nyström

Humanities Lab, Lund University

Richard Dewhurst

Interacting Minds Centre, Århus University

Mikael Johansson

Lund University https://orcid.org/0000-0002-2903-6301

\section{Article}

Keywords: eye-movement replay, visuospatial relations, episodic remembering

Posted Date: February 25th, 2021

DOI: https://doi.org/10.21203/rs.3.rs-275969/v1

License: (c) (1) This work is licensed under a Creative Commons Attribution 4.0 International License. Read Full License 
Running head: EYE-MOVEMENT REPLAY SUPPORTS EPISODIC REMEMBERING

\section{Eye-movement replay supports episodic remembering}

2

3 Roger Johansson ${ }^{1 *}$, Marcus Nyström² ${ }^{2}$, Richard Dewhurst ${ }^{3} \&$ Mikael Johansson $^{1}$

$4 \quad{ }^{1}$ Dept. of Psychology, Lund University, Sweden

$5 \quad{ }^{2}$ Humanities Lab, Lund University, Sweden

$6 \quad$ Interacting Minds Centre, Århus University, Denmark

7

8

9

10

Author note

11

* Correspondence concerning this article should be addressed to Roger Johansson,

12

Department of Psychology, Lund University, Box 213, SE-221 00 Lund, Sweden, email:

$13 \quad$ roger.johansson@psy.lu.se 


\section{Abstract}

2 When we bring to mind something we have seen before, our eyes spontaneously reproduce a 3 pattern strikingly similar to that made during the original encounter. Eye-movements can then

4 serve the opposite purpose to acquiring new visual information; they can serve as self-generated 5 cues, pointing to memories already stored. By isolating separable properties within the closely

6 bound chain of where and when we look, we demonstrate that specific components of 7 dynamically reinstated eye-movement sequences, facilitate different aspects of episodic 8 remembering. We also show that the fidelity with which a series of connected eye-movements 9 from initial encoding is reproduced during subsequent retrieval, predicts the quality of the 10 recalled memory. Our findings indicate that eye movements are "replayed" to assemble 11 visuospatial relations as we remember. Distinct dimensions of these scanpaths differentially 12 contribute depending on the goal-relevant memory. 


\section{Eye-movement replay supports episodic remembering}

2 Episodic memory refers to memories of events that we have experienced in a specific time and

3 place, allowing us to relive previous events with a considerable level of detail and specificity ${ }^{1}$.

4 The formation of coherent episodic memories critically hinges upon the binding of 5 spatiotemporal relationships into a context, which largely depends on how we visually "sample"

6 the world when we act upon it via eye movements ${ }^{2}$. Although only a limited amount of 7 information is processed at each sample, the sequence of eye movements effectively binds inter8 related episodic details together, allowing us to encode a memory representation of the event 9 as a whole ${ }^{3,4}$. Thus, our visual sampling of the world is highly predictive of the content and quality of episodic memory formation ${ }^{5-11}$. Spontaneous eye movements also occur during retrieval of episodic memories, even when there is no longer any relevant information present. These have been demonstrated to largely reproduce the gaze patterns that were established during encoding ${ }^{12-18}$. A prominent view holds that such gaze reinstatements actively support episodic remembering ${ }^{19-21}$, and that eye movements may serve to assemble and organize visuospatial relations across time and space into coherent memories ${ }^{12,13,21-25}$. This has never been directly demonstrated, however.

The claim that eye movements have a functional role during episodic remembering receives support from previous research showing that the extent of encoding-retrieval overlaps in fixation locations positively correlates with retrieval performance ${ }^{13,26,27}$, and with neural reactivation during retrieval ${ }^{28,29}$. Extending such findings, it has been demonstrated that the likelihood of successful remembering increases when gaze locations during recall are directly manipulated to overlap with those from encoding ${ }^{30-34}$. While these findings clearly show that where you look has important consequences for what you remember, they are all related to mere gaze positions, and do not take into account how eye-movements are connected and unfold over 
1 time. Also, active manipulations of gaze direction eliminate the spatiotemporal dynamics that

2 are characteristic of free viewing ${ }^{35}$. To date, there is no evidence for the influential claim that eye-movement reinstatement serves to facilitate episodic reconstruction by binding the constituent parts of the recalled event together into its original spatiotemporal context ${ }^{12,16,21-26}$. To address this fundamental question, it is necessary to go beyond static gaze positions alone and examine how the replay of whole sequences of connected eye movements - scanpaths may support episodic remembering.

In the present study, we tackle this issue head on by utilizing state-of-the-art scanpath similarity techniques, capable of decomposing the encoding-retrieval overlap of scanpaths into distinct spatiotemporal properties. It is then possible to examine the degree to which these properties become reinstated during episodic retrieval. Participants encode 36 images with associated verbal labels and subsequently recall each image while looking at a blank screen. In the recall phase, a particular image is cued by its verbal label, and the quality of each episodic reconstruction is rated with respect to overall memory, vividness and spatial accuracy (Fig. 1B). To determine if individual scanpath properties objectively predict the strength and quality of the mnemonic content during recall, our paradigm further combines those subjective ratings of memory quality with a subsequent surprise forced-choice recognition test (Fig. 1B). Finally, we use two types of image stimuli - scenes and object arrangements - to investigate if the individual scanpath properties differentially contribute depending on the nature of the goalrelevant memory. The scenes $(\mathrm{N}=18)$ comprise highly predictable spatial relations between meaningful scene elements, with relatively low demands on relational memory, whereas the object arrangements $(\mathrm{N}=18)$ comprise arbitrary spatial relations between their scene elements (objects), with relatively high demands on relational memory (Fig. 1A). These two types of 
1 stimuli allow us to examine the relative contribution of different scanpath reinstatements as a

2 function of varying demands on relational memory ${ }^{21}$.

A. Stimuli examples

Scenes

3

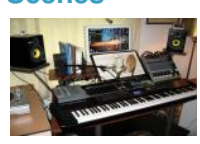

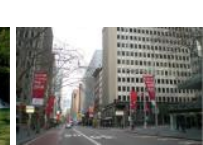

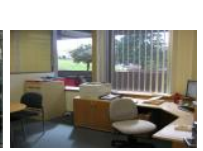

Object arrangements
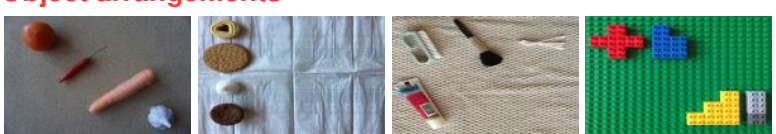

\section{B. Experimental design}

\section{Encoding}
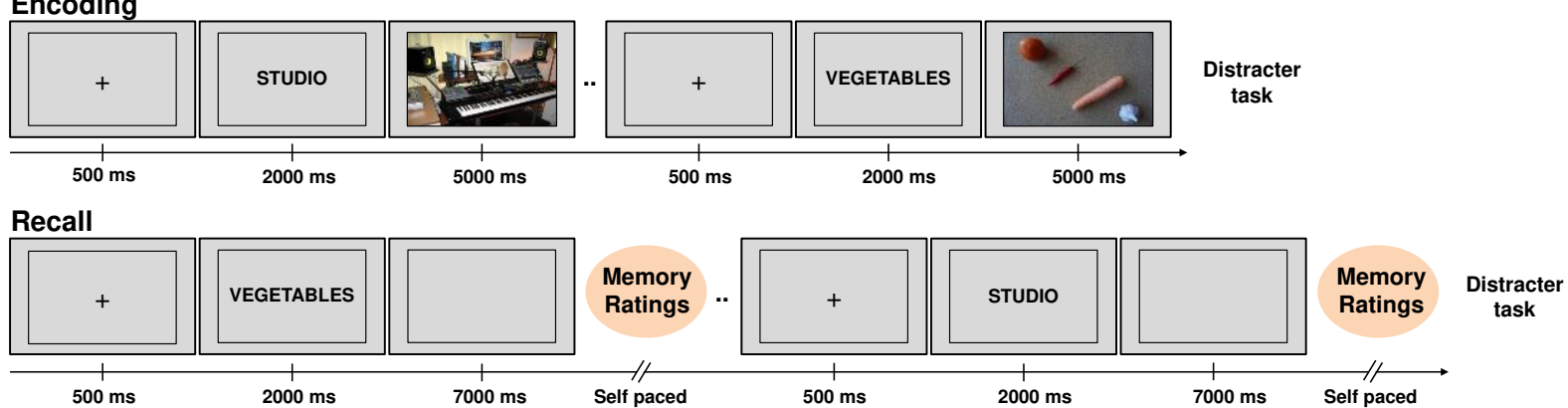

Surprise test: 4AFC Recognition test

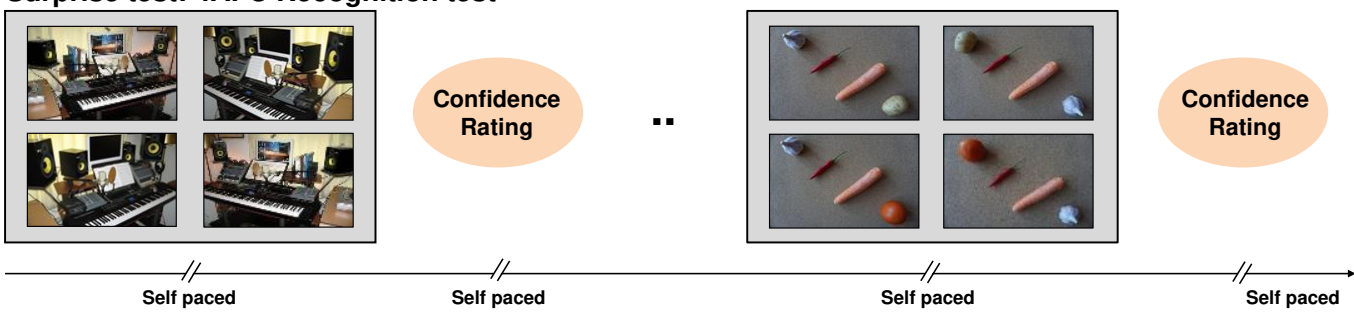

4

\section{Results}




\section{Behavioral results}

2 We first sought to verify that the object arrangements were more demanding to recall than the

3 scenes. As expected, mnemonic content was rated higher for scenes than object arrangements

4 during recall, $\beta=0.165,(S E=0.0317), z=5.22, p<.001$. For the surprise test, results

5 revealed higher performance for scenes over four different measures of retrieval performance:

6 response accuracy, $\chi^{2}(1)=64.8, \beta=2.17,(S E=0.269), z=8.05, p<.001 ;$ confidence, $\chi^{2}(1)=$

$751.0, \beta=0.175,(S E=0.0245), z=7.14, p<.001 ;$ response time, $\chi^{2}(1)=43.7, \beta=4132,(S E$

$8=625), z=6.61, p<.001$; gaze transitions between options (a measure of choice certainty ${ }^{36}$ ),

$9 \chi^{2}(1)=53.6, \beta=4.62,(S E=0.631), z=7.32, p<.001$. Thus, these results demonstrate that the object arrangements, placing greater demand on relational memory, were indeed harder to

11 recall than the scenes (see Table 1).

Table 1 Object arrangements are more demanding to recall than scenes. Mean values for the performance data during the recall phase and the surprise test, with standard deviations within brackets. The mnemonic content score represents the mean of the three subjective ratings (overall memory, vividness, spatial accuracy). Only correct trials were considered for confidence, response time and gaze transitions between options (a measure of choice certainty $\left.{ }^{36}\right)$.

\begin{tabular}{lccc}
\hline & Total & Scenes & Object arrangements \\
\hline $\begin{array}{l}\text { Performance data } \\
\text { Recall phase }\end{array}$ & & & \\
Mnemonic Content Score (\%) & $58(30)$ & $66(26)$ & $49(31)$ \\
& & & \\
Surprise test & & & \\
Accuracy (\%) & $85(36)$ & $95(22)$ & $75(43)$ \\
Response time (ms) & $7699(6746)$ & $5583(5672)$ & $9815(7065)$ \\
Confidence (\%) & $78(29)$ & $90(18)$ & $66(33)$ \\
Gaze transitions between options & $10.8(7.4)$ & $8.5(5.2)$ & $13.2(8.4)$ \\
& & & \\
\hline
\end{tabular}


1 We next tested if the mnemonic content score during recall predicted subsequent retrieval

2 performance during the surprise test. Results reveal that the mnemonic content score was a

3 significant predictor of all four measures of retrieval performance: response accuracy, $\chi^{2}(1)=$

$4 \quad 6.17, \beta=0.645,(S E=0.260), z=2.48, p=.013$; confidence, $\chi^{2}(1)=27.4, \beta=0.101,(S E=$

$50.0192), z=5.24, p<.001$; response time, $\chi^{2}(1)=6.53, \beta=-1339,(S E=524), z=-2.55, p=$

6.011 ; choice certainty, $\chi^{2}(1)=11.8, \beta=-1.98,(S E=0.575), z=-3.44, p<.001$. See Fig. 2A-D.

7 Higher subjective ratings during the recall phase thus predicted more accurate, confident, faster

8 and certain responses during the subsequent surprise test. We therefore conclude that the

9 mnemonic content score during recall is a representative index of the memory quality during episodic remembering.
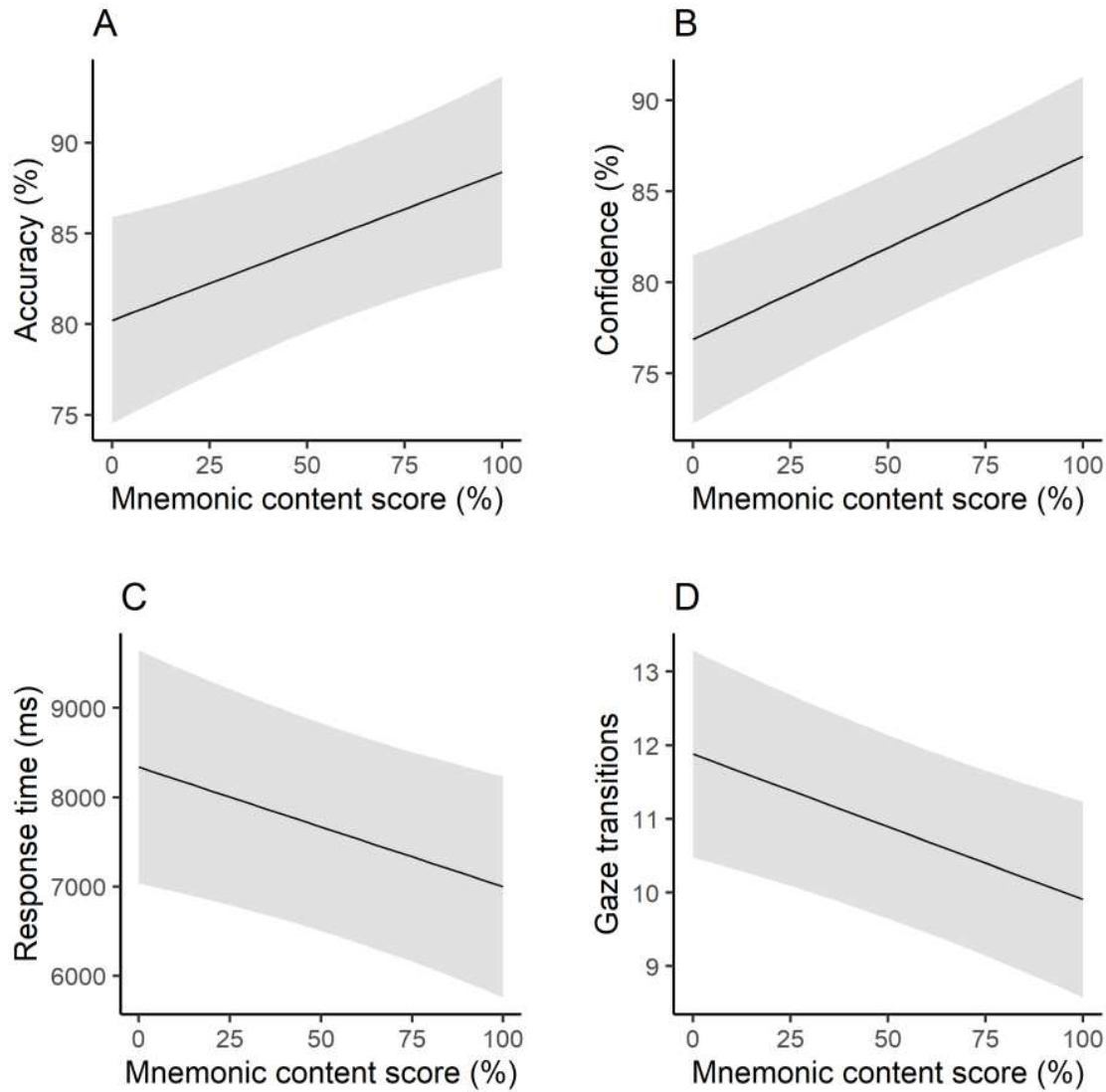

Fig 2 Memory quality during recall predicts subsequent recognition. How the mnemonic content score from the recall phase predicted (A) accuracy, (B) confidence, (C) response time, and (D) choice certainty in the corresponding 4AFC recognition task. Error bars and shaded areas denote $95 \%$ Confidence Intervals. 


\section{Reinstatement of gaze positions during episodic remembering}

3 In the next step, we examined reinstatement of static position-based gaze, i.e., without

4 considering the temporal succession of eye movements. Here, we found significant encoding-

5 retrieval-overlap, and this effect was most pronounced for the more demanding object arrangements (Fig. 3A). Importantly, we also found that the degree of this reinstatement of gaze positions predicted the quality of episodic remembering (Fig. 3B). This corroborates

8 what previous research has found ${ }^{13,26,27}$, and provide further evidence that the reinstatement of 9 gaze positions supports episodic remembering. An exhaustive presentation of this analysis is available as Supplementary material (supplementary details on results).

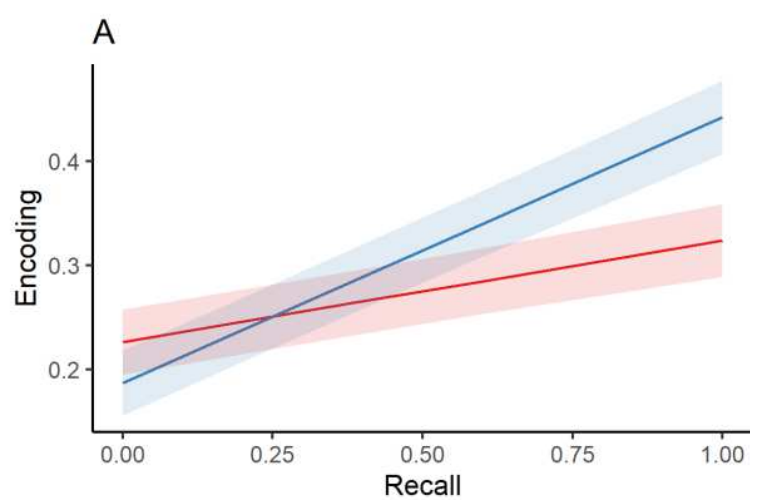

Scenes

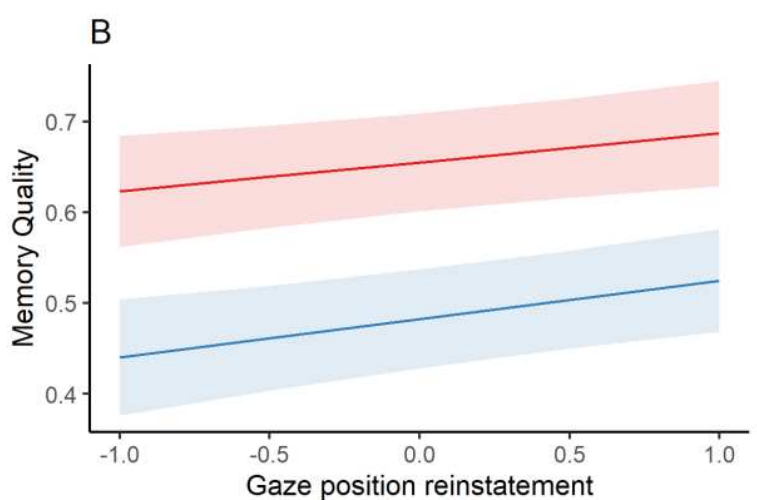

Object arrangements
11

\section{Scanpath reinstatement during episodic remembering}


1 To achieve the central goals of the present study, we next investigated encoding-retrieval

2 overlap in scanpaths, and how the reinstatement of dynamically unfolding eye movements

3 may support episodic remembering.

4

5 To measure scanpath similarity between encoding and recall, we used MultiMatch (MM),

6 which was introduced as a method for comparing scanpaths over different spatiotemporal

7 dimensions ${ }^{37}$, and has subsequently been validated, applied, and evaluated against other

8 scanpath comparison tools ${ }^{35,38-41}$. The basic principle is that the MM algorithm simplifies the

9 two scanpaths under comparison (in this case, one from encoding and the other from recall)

into virtualized sequences of saccadic vectors and then temporally aligns them with each

other. Similarity between the temporally aligned scanpaths can then be separately determined

12 for the MM-dimensions: (1) position, (2) shape, (3) direction, (4) length, and (5) duration. See

Fig. 4A. The five MM-dimensions of scanpath similarity thus reflect different embodied

\section{A. Scanpath Similarity analysis (MultiMatch)}

Scanpath 1
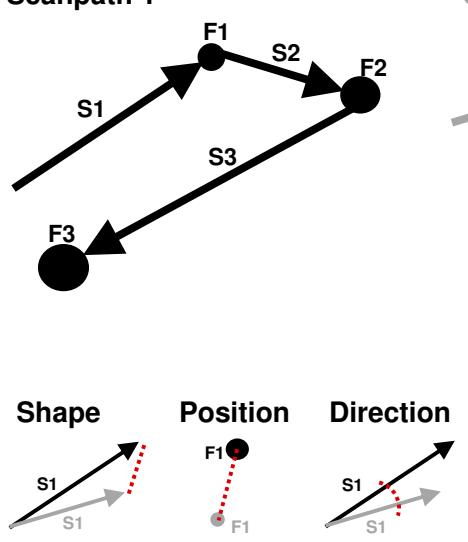

\section{Scanpath 2}

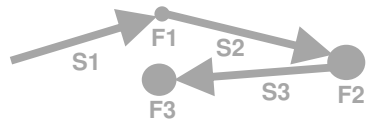

2 i.
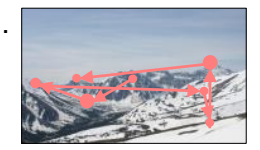

iii.
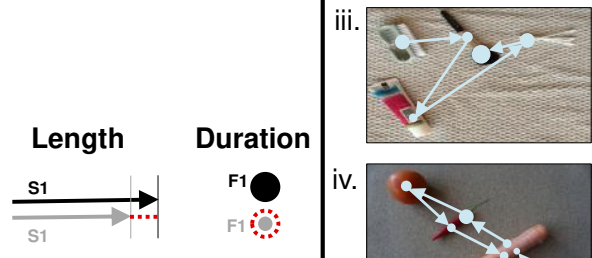

B. Examples of varying Encoding-Retrieval similiarity Encoding

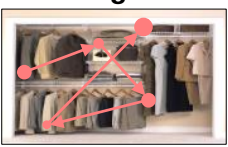
Recall

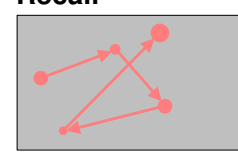
High Position High Shape High Direction High Length High Duration

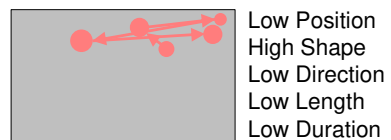
Low Duration

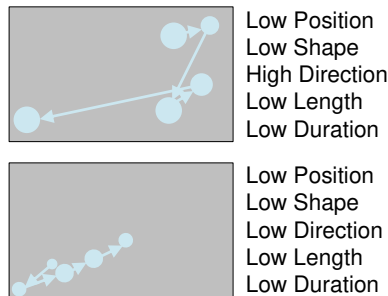


1 Fig 4 Scanpath similarity. (A) Overview of the MultiMatch scanpath similarity analysis. The top panel shows two relatively similar scanpaths under comparison, where fixations are represented as dots (F1-F3) and saccades as arrows between the fixations (S1-S3). Larger dots represent longer fixation duration. The bottom panel illustrates differences over the five dimensions of shape, position, direction, length and duration for the first pairs of aligned saccades (S1) and fixations (F1). The numeric difference between each dimension is illustrated with a red dotted line for each dimension separately. The Position dimension relies on spatial coordinates in absolute space and quantifies how similar aligned fixations are in respect to Euclidean distances. In contrast, the Shape, Direction and Length dimensions rely on differences in relative space. The Shape dimension quantifies how similar aligned saccadic vectors are in overall geometric shape, whereas the Direction dimension quantifies how similar aligned saccadic vectors are in geometric angle. The Length dimension quantifies how similar aligned saccades are in their absolute amplitude. The Duration dimension does not rely on any spatial coordinates and quantifies how similar aligned fixations are in their duration.

(B) Examples of varying encoding-retrieval similarity: (i) complete encoding-retrieval overlap in respect to all five MM-dimensions; (ii) relatively high encoding-retrieval similarity in Shape, but relatively low similarity in Position (due to dislocation in absolute space during recall), Direction (due to large differences in saccadic angles during recall), Length (due to overall shorter saccades during recall), and Duration (due to overall longer fixation durations during recall); (iii) high encoding-retrieval similarity in Direction, but low similarity in Shape (due to local differences in saccadic lengths during recall), Position (due to dislocation in absolute space during recall), Length (due to disproportional saccadic lengths during recall), and Duration (due to overall longer fixation durations during recall); (iv) low similarity over all five MM-dimensions.

To determine if and how scanpaths from encoding become reinstated during recall, we first calculated encoding-retrieval-similarity scores over all five MM-dimensions (Position, Shape, Direction, Length, and Duration) by comparing the scanpaths produced by a participant during the encoding of an image, with those produced during recall of the same image. See Fig. 4B for examples of varying encoding-retrieval similarity over the five MM-dimensions. Second, by comparing the scanpaths produced by a participant during the encoding of an image with those produced during recall of all the other images from the same type (Scenes, 
1 Object arrangements), and then averaging across the scores calculated for all images, we

2 acquired a Baseline Similarity score. This represents the average encoding-retrieval similarity

3 in scanpaths for each participant across images.

4

5 For the scenes, results revealed that the encoding-retrieval similarity score was significantly

6 higher than the baseline similarity score for position, $\chi^{2}(1)=6.83, \beta=0.0048,(S E=0.0018)$,

$7 z=2.61, p=.009$, and shape, $\chi^{2}(1)=5.70, \beta=0.0030,(S E=0.0013), z=2.39, p=.017$. For

8 the object arrangements, results revealed that the encoding-retrieval similarity score was

9 significantly higher than the baseline similarity score for position, $\chi^{2}(1)=148, \beta=0.0278$, $(S E=0.0023), z=12.2, p<.001$, shape, $\chi^{2}(1)=55.8, \beta=0.0106,(S E=0.0014), z=7.47, p<$ .001 , and direction, $\chi^{2}(1)=33.0, \beta=0.0269,(S E=0.0047), z=5.74, p<.001$. Length and duration were not significant predictors for either scenes or object arrangements $\left(\chi^{2}(1)<2.30\right.$, $p s>.13)$. See Fig. 5A-B. These results thus show that position, shape and direction are scanpath properties that were reinstated during recall. However, direction reinstatement was only observed for the object arrangements. Length and duration were not reinstated for either case of image type. As the length and duration dimensions typically depend on visual properties from the image $\mathrm{e}^{40,42}$, which are absent during recall, there is no particular reason to expect encoding-retrieval similarity for either of those. Moreover, it is well known that fixation durations become longer when there are no external features to look at ${ }^{12}$. 

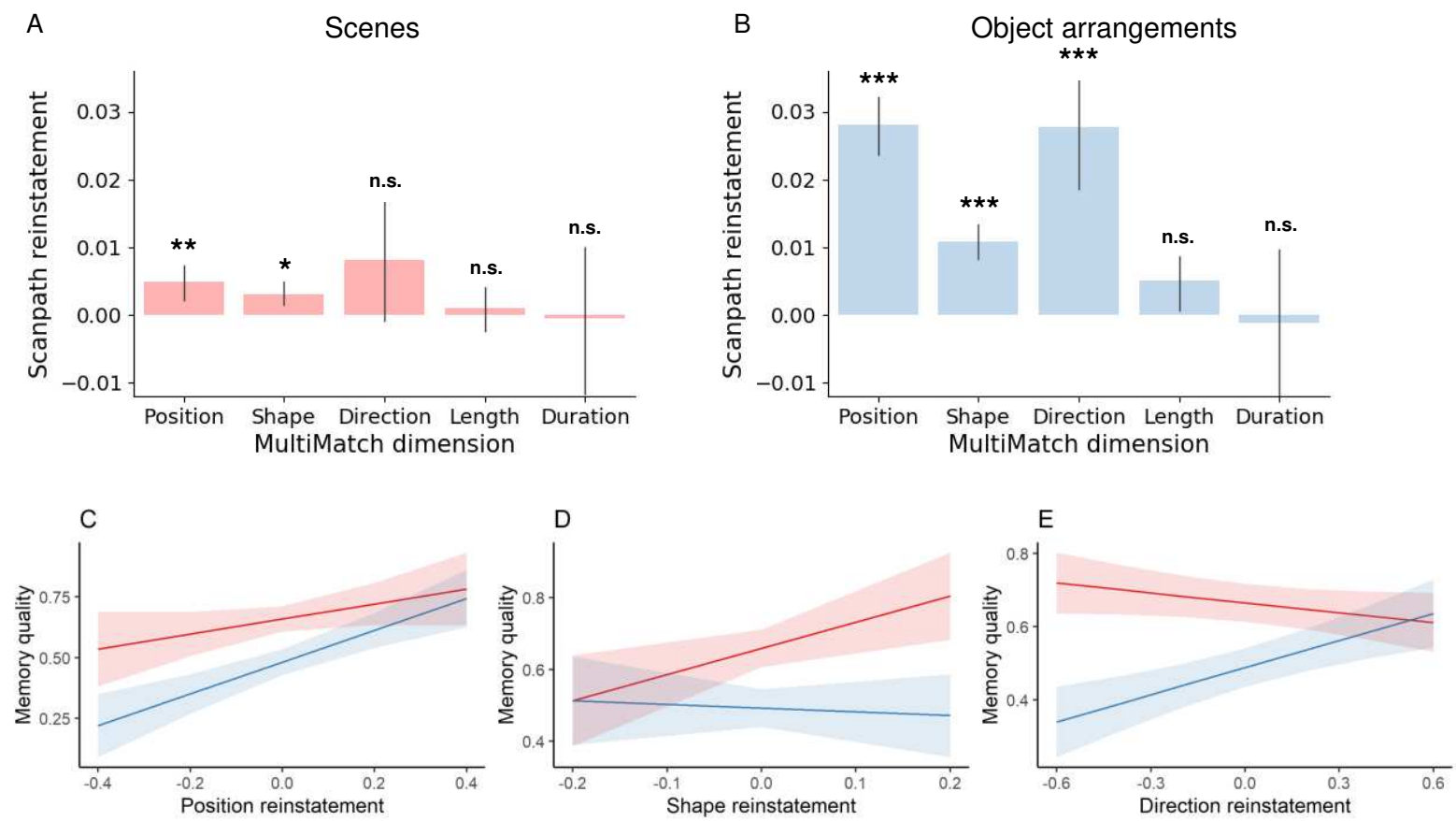

Scenes

Object arrangements

$\frac{1}{2}$

3

4

5

6

7

8

\section{Functional relevance for episodic remembering}

In the final step, we then tested whether the degree of scanpath reinstatement for the significant MM-dimensions (position, shape and direction) predicted the quality of episodic remembering during the recall phase. To quantify the degree of scanpath reinstatement, we used a similar rationale as Wynn, Ryan and Buchsbaum ${ }^{43}$, and subtracted the Baseline Similarity score from the Encoding-Retrieval-Similarity score for each MM-dimension over each participant and image. The best model fit of memory quality revealed significant effects of image type, position 


\section{EYE-MOVEMENT REPLAY SUPPORTS EPISODIC REMEMBERING}

1 reinstatement, and significant interaction effects between image type and shape reinstatement,

2 and between image type and direction reinstatement (Table 2). Greater position reinstatement

3 predicted better memory quality independent of image type (Fig. 5C), whereas greater shape

4 reinstatement only predicted better memory quality for the scenes (Fig. 5D), and greater

5 direction reinstatement only predicted better memory quality for the object arrangements (Fig.

$6 \quad 5 \mathrm{E})$.

7

8 Table 2 Scanpath reinstatement predicts memory quality. Results of the best fit model of Memory Quality,

9 with Image Type (Scenes, Object arrangements), and the significant MultiMatch dimensions (Shape, Position,

Direction) as the independent variables. Participants and Images were modelled as random effects (intercepts).

R-squared conditional $=0.37$.

\begin{tabular}{|c|c|c|c|c|c|}
\hline Effects & $B$ & $S E$ & $95 \% C I$ & $t$ & $p$ \\
\hline (Intercept) & 0.578 & 0.022 & $0.533,0.621$ & 25.91 & $<.001 * * *$ \\
\hline Image Type & 0.172 & 0.030 & $0.114,0.229$ & 5.83 & $<.001 * * *$ \\
\hline Shape Reinstatement & 0.312 & 0.197 & $-0.075,0.699$ & 1.58 & 0.114 \\
\hline Position Reinstatement & 0.480 & 0.116 & $0.254,0.707$ & 4.16 & $<.001 * * *$ \\
\hline Direction Reinstatement & 0.079 & 0.043 & $-0.005,0.162$ & 1.84 & 0.066 \\
\hline Image Type $\times$ Shape Reinstatement & 0.831 & 0.395 & $0.056,1.606$ & 2.10 & $0.036 *$ \\
\hline Image Type $\times$ Position Reinstatement & -0.345 & 0.231 & $-0.798,0.109$ & -1.49 & 0.136 \\
\hline \multirow[t]{2}{*}{ Image Type $\times$ Direction Reinstatement } & -0.336 & 0.085 & $-0.504,-0.169$ & -3.94 & $<.001 * * *$ \\
\hline & & Variance & SD & & \\
\hline Random effects for Participant (Intercept) & & 0.0168 & 0.130 & & \\
\hline Random effects for Image (Intercept) & & 0.007 & 0.082 & & \\
\hline
\end{tabular}

Model equation: Memory Quality $\sim 1+$ Image Type + Shape Reinstatement+ Position Reinstatement +

Direction Reinstatement + Image Type $\times$ Shape Reinstatement + Image Type $\times$ Position Reinstatement

+ Image Type $\times$ Direction Reinstatement $+(1 \mid \mathrm{FP})+(1 \mid$ Image $)$

Total observations: 2017

12

13 The results thus show that independent of the mnemonic content, looking at locations (i.e.

14 position reinstatement) in a similar order during retrieval as during the original encoding 
1 predicts the quality of episodic remembering. The interaction effects demonstrate that shape

2 and direction reinstatement contributed differently depending on the mnemonic content.

3 General spatial structure (i.e. shape reinstatement) appears to be more important when

4 reconstructing the more predictable spatial relations in the scene images. Conversly, the

5 particular heading of eye movements (i.e. direction reinstatement) appears to be more important

6 when reconstructing the arbitrary spatial relations in the object arrangements.

\section{Facilitating remembering or a consequence of the memory representation?}

While the results indicate a functional role of scanpath reinstatements in episodic reconstruction, an alternative explanation is that greater reinstatement is a consequence of having a strong memory representation. To disambiguate between those two explanations, we sought to determine whether scanpath reinstatements depends on how well the images are initially encoded into memory. Previous research has established that the cumulative number of fixations during encoding is a reliable index of how well a visual stimulus is encoded into memory ${ }^{5-11}$. Thus, to account for effects of encoding strength, we added the cumulative number of study fixations on each image into our model. The model was significantly improved, $\chi^{2}(1)=24.90, p<.001$. All previous effects and interaction effects were significant also in this model, and as expected, the cumulative number of study fixations significantly predicted memory quality during recall, $\beta=0.008,(S E=0.002), z=4.99, p<.001$, which confirms the validity of this measure as an index of encoding strength. Interactions of number of study fixations and the other predictors (position reinstatement, shape reinstatement, direction reinstatement, image type), were then stepwise added to the model. Importantly, none of those interactions significantly improved the model ( $p$-values $>.19)$. Thus, the reported effects of scanpath reinstatements upon memory quality do not reduce to how well the images were originally encoded into memory. Instead, our results substantiate the idea that 
1 the replay of eye movements supports the reconstruction of inter-related and task-relevant

2 mnemonic features into a spatiotemporal context.

\section{Discussion}

5 Corroborating previous research ${ }^{13,2627,30,31}$ we found that encoding-retrieval similarity in gaze locations predicts the fidelity of episodic remembering. State-of-the-art scanpath-similarity techniques (MultiMatch ${ }^{35}$ ) allowed us to move beyond this to examine reinstatement of qualitatively different scanpath properties during episodic reconstructions. Of central importance, we provide evidence that the replay of eye movement behavior, over-and-above static position-based gaze, supports episodic remembering and that different spatiotemporal properties of the unfolding scanpaths differentially contribute depending on the nature of the goal-relevant memory. Specifically, we found that encoding-retrieval overlaps in scanpath shape contribute more to reconstructing the memory of a global scene structure; whereas encoding-retrieval overlaps in scanpath direction contribute more to reconstructing particular object arrangements. Finally, we provide evidence that these scanpath reinstatement effects on episodic remembering are not simply a consequence of how well the event was originally encoded.

The claim that gaze behavior actively supports episodic recollection dates back to (at least) Ulric Neisser and Donald Hebb. Neisser argued that eye movements - or the processes that drives them - are actively associated with the construction of a visual memory ${ }^{23}$, and Hebb claimed that eye movements are necessary to assemble and organize "part images" into a whole visualized image ${ }^{22}$. Such ideas were further developed in Noton and Stark's Scanpath Theory, which states that memories are stored in a "feature ring", comprising visual features and the sequence of eye movements (scanpaths) linking them together ${ }^{24,25}$. Scanpath Theory holds that 
1 (1) eye movements during recall play out in the same sequential order as during encoding, and

2 (2) such scanpath replay serves a functional role in episodic remembering. While a strong interpretation of Scanpath Theory, where memory is accompanied by an exact and full scanpath recapitulation, has been refuted in more recent research ${ }^{16,21,44}$, there is extensive evidence that episodic remembering involves eye movements that largely reproduce gaze patterns at encoding ${ }^{12-16,26,28}$. Thus, the first tenet of Scanpath Theory, that episodic remembering is accompanied by scanpath replay, is well established in the literature, and gains further support from the present study. However, there is to date virtually no evidence for the second tenet, that scanpath reinstatements play a functional role in episodic memory retrieval. Recent work has demonstrated that gaze reinstatements promote cortical reconstruction ${ }^{28,29}$ and successful remembering ${ }^{13,26,27,30-34}$. However, these findings are all related to static position-based gaze and shed no light upon the proposition that unfolding sequences of connected eye movements reconstruct and bind spatiotemporal information into a coherent memory. Interesting recent research on recognition memory shows that a temporal replay of gaze patterns can support recognition performance ${ }^{45,46}$. Still, recognition tasks involve situations where all encoded information is available as a "copy cue", and where visual information accumulates until sufficient evidence is available to solve the old/new-discrimination task. The purpose of visual exploration under such conditions is thus considerably different from a recall task, where the rememberer needs to mentally reconstruct the complete spatiotemporal properties of a prior event without supporting visual input ${ }^{5}$. Here, we present direct evidence that a temporal replay of eye movements plays an active role in episodic reconstruction in a pure recall task.

Recently, Wynn and colleagues proposed that eye movements support active memory retrieval by broadly reinstating the spatiotemporal context based on current task demands and available cognitive resources $^{21}$. The idea that the facilitatory role of gaze reinstatements increases with 
1 task demands has been supported by their studies on older adults ${ }^{27}$ and task difficulty ${ }^{43}$. Further

2 support comes from the present findings where more extensive scanpath reinstatements where seen during the reconstruction of the object arrangements as compared to the scenes. The scenes comprised predictable spatial relations between meaningful elements due to schematic and situational knowledge for the common scenarios, and memory for such information poses relatively low demands on relational memory. Reconstruction of such an event should involve a rather coarse arrangement of key elements within the overall context, which was also supported by the finding that the general spatial structure of the scanpath (position and shape) contributed to successful remembering. The object arrangements, on the other hand, comprised arbitrary spatial relations among scene elements (objects), which puts relatively high demands on relational memory. The ensuing reconstruction should require a more precise arranging of the objects into an ordered context, which was supported by the finding that the scanpath properties of position and direction contributed to successful remembering.

A vast amount of research has demonstrated that the hippocampus plays a critical role in binding information in space and time during memory formation, and it is assumed that the hippocampus stores an event index, pointing to the cortical representations of each aspect of the whole event (for a review see ref. ${ }^{47}$ ). Remembering depends on a retrieval cue matching the hippocampal index, which in turn triggers pattern completion and the reactivation of the cortical traces (and thus the experience of remembering the original event). Based on this cortical-hippocampal interplay, a prominent view holds that the reconstruction of past events fundamentally relies on mental simulations that reinstate approximations of the sensorimotor processes that characterized the original event ${ }^{48,49}$. Successful episodic remembering is considered to depend on the overlap between available retrieval cues and stored memory traces $^{50}$. Compatibility between the processes triggered by a retrieval cue and those engaged 
1 during encoding increases the likelihood of successful retrieval ${ }^{50,51}$. As previous research has

2 demonstrated that gaze behavior during encoding and retrieval are linked to activity in the

3 hippocampus ${ }^{52-55}$, and that gaze reinstatement correlates with neural reactivation ${ }^{28,29}$, it is

4 conceivable that the scanpath replay reported here is responsible for promoting cortical-

5 hippocampal reactivation of visuospatial relations during episodic memory reconstruction. The

6 successive reinstatement of eye movements may act as internally generated retrieval cues that

7 continuously updates in an iterative fashion as the rememberer moves his/her eyes during the

8 pattern completion process. In situations with high demands on relational memory, as for the

9 recollection of the object arrangements, more specified retrieval cues would be required during the pattern completion process, as compared to the less demanding scenes, where the pattern completion process would be supported by more predictable scene semantics.

\section{Conclusion}

Reinstatement of gaze positions has proven important for episodic remembering. The present study extends this finding and provides direct evidence that the actual replay of eye movements unfolding over time facilitates episodic remembering, and that specific spatiotemporal scanpath properties differentially contribute depending on the nature of the goal-relevant memory.

\section{Methods}

\section{Participants}

Sixty-two healthy adults participated in the experiment. Two were removed owing to extensive data loss and technical problems, leaving sixty participants (34 females; mean age 25.2, SD 6.4). Power analyses ${ }^{56}$ for bivariate measures of association indicated that our sample size should be sufficient to detect a large correlation $(\mathrm{r}=0.5)$ and difference between means $(d=.6)$ with $90 \%$ power $(\alpha=0.05)$, which is in line with prior work on gaze 
1 reinstatement ${ }^{16,26}$ and scanpath similarity ${ }^{39}$. All participants were fluent in Swedish and had

2 normal or corrected-to-normal vision. Participants gave written informed consents and were compensated with a cinema voucher. All methods were conducted in accordance with the Swedish Act concerning the Ethical Review of Research involving Humans (2003:460).

\section{Data Acquisition and Materials}

Gaze data was recorded from both eyes individually, using a Sensory Motoric Instruments (SMI RED-M) eye tracker, running iView X 2.7 software and sampling at $120 \mathrm{~Hz}$. A Dell Optiplex 755 PC presented stimuli using PsychoPy ${ }^{57}$ on a 22' ' monitor with a resolution of $1680 \times 1050$ pixels. Participants were seated with their heads in a chin and forehead rest, 65 $\mathrm{cm}$ away from the monitor. Calibration and validation of gaze data was conducted prior to each participant's experimental session. Spatial accuracy during validation across all participants was $0.53 \pm 0.22$ degrees (x offset), and 0.48 \pm 0.22 (y offset) $(\mathrm{M} \pm \mathrm{SD})$. Spatial precision was computed for each participant as the root-median-square (RMS) of inter-sample distances for all recorded samples. The average RMS value across all participants was $0.10 \pm$ 0.19 degrees (x offset), and $0.08 \pm 0.15$ degrees (y offset) $(\mathrm{M} \pm \mathrm{SD})$. Proportion data loss was defined as all lost samples divided by the total number of samples. Proportion data lost across all participants was $0.05 \pm 0.14(\mathrm{M} \pm \mathrm{SD})$. Fixations were detected with the $\mathrm{I} 2 \mathrm{MC}$ algorithm ${ }^{58}$. Stimuli comprised 18 scenes and 18 object arrangements that were presented in a frame that shot for this particular study (available here: https://osf.io/d9zng/).

The content of the scene images comprised an equal amount of indoor and outdoor global 
1 the image's semantic structure. The content of the object arrangement images comprised four

2 separate objects from the same category, where the spatial relations between the objects were completely arbitrary in respect to the image's semantic structure. The 18 object arrangements all comprised unique spatial configurations (see Fig. 1A; complete set of images available here: https://osf.io/d9zng/). In contrast to the scene images, we thus had full experimental control over the spatial relations among individual scene elements (objects) in the object arrangement images.

\section{Design and procedure}

The experiment was divided into three phases: encoding, recall, and surprise test (see Fig. 1B). To conceal the true objective of the study, participants were told that the experiment concerned pupil dilation in relation to mental workload, and it was explained that their eyes were filmed for this matter.

Encoding phase. Participants encoded 36 images accompanied by a verbal label that preceded each image. The verbal label described the semantic content of the succeeding image (e.g., "studio", "vegetables"). The images comprised two different types: scenes (n= 18) and object arrangements $(\mathrm{n}=18)$ and were each presented for $5 \mathrm{~s}$ in randomized order. Participants were instructed to memorize each image as thoroughly as possible (see Fig. 1A). When the encoding phase finished, participants engaged in a distracter task, where they were to count backwards in steps of 3 from a randomly generated three-digit number for $12 \mathrm{~s}$.

Recall phase. Participants recalled all 36 encoded images while looking at a blank screen. Participants were cued by the associated verbal label (preceding the blank screen) in a randomized order and were then instructed to recall and visualize the corresponding image in 
as much detail as possible while looking at the blank screen. The blank screen remained for 7 s. After each recollection, participants were to rate the quality of their recollection based on overall memory, vividness and spatial accuracy. Overall memory was rated on a scale $0-100 \%$ in relation to the statement "It was easy for me to remember the image". Vividness was rated on a scale $0-100 \%$ in relation to the statement "My mental image was clear, vivid and detailed - almost as if I could see the image in front of me". Spatial accuracy was rated on a scale 0$100 \%$ in relation to the statement "I could indicate, with high spatial accuracy, where different objects/scene elements were located in the image". See Table 1. Based on a mean of those three ratings, a Mnemonic Content Score was calculated for each image and participant.

Surprise test. Participants completed four-alternative forced-choice (4AFC) recognition tests that covered all 36 images presented during encoding. Participants were not aware that they would engage in this test beforehand. The target image and three different distracter images were presented in the four quadrants of the screen, and participants were instructed to select the image they had encoded and recalled as accurately and quickly as possible. After each selection, participants rated how confident $(0-100 \%)$ they were in their choice. For the scenes, the distracter images comprised: (1) a horizontal flip of the target image; (2) a similar lure image; and (3) a horizontal flip of the lure image. For the object arrangements, the distracter images comprised: (1) a lure image, where two of the original objects had switched locations; (2) a lure image, where one of the objects had been exchanged with another semantically congruent object; and (3) a lure image, where one of the objects had been exchanged with the other semantically congruent object, and also the location of this new object had been switched with one of the other objects. 
1 The present experimental design allowed us to collect spontaneous eye movements during

2 recall, and to use the surprise test to evaluate the validity of the subjective ratings as a

3 measure of recall success.

4

5

6

7

\section{Author contributions}

R.J. and M.J. conceived the study. R.J. designed the study. R.J., M.N., and R.D. implemented and conducted the experiment. R.J. and M.N. analyzed the data. R.J and M.J. wrote the manuscript, with comments and edits by R.D. and M.N.

\section{Acknowledgments}

We gratefully acknowledge Lund University Humanities Lab. The work was funded by the Swedish Research Council grant no. 2015-01206 (Roger Johansson) and the Marcus and Amalia Wallenberg Foundation award MAW2015.0043 (Mikael Johansson). 
EYE-MOVEMENT REPLAY SUPPORTS EPISODIC REMEMBERING

1

2 Additional information

3 Supplementary information includes supplementary details on results

4 Correspondence and requests for materials should be addressed to R.J.

5 Competing interests

6 The authors declare no competing interests 
EYE-MOVEMENT REPLAY SUPPORTS EPISODIC REMEMBERING

\section{$1 \quad$ References}

2 1. Tulving, E. Elements of Episodic Memory (Clarendon Press, 1983).

3 2. Voss, J. L., Bridge, D. J., Cohen, N. J., \& Walker, J. A. A closer look at the hippocampus and memory. Trends Cogn. Sci. 21, 577-588 (2017).

3. Findlay, J. M., Findlay, J. M., \& Gilchrist, I. D. Active vision: The psychology of looking and seeing (Oxford University Press, 2003).

4. Henderson, J. M. Human gaze control during real-world scene perception. Trends Cogn. Sci. 7, 498-504 (2003).

5. Damiano, C., \& Walther, D. B. Distinct roles of eye movements during memory encoding and retrieval. Cognition 184, 119-129 (2019).

6. Fehlmann, B., Coynel, D., Schicktanz, N., Milnik, A., Gschwind, L., Hofmann, P.,

8. Liu, Z. X., Shen, K., Olsen, R. K., \& Ryan, J. D. Visual sampling predicts hippocampal activity. J. Neurosci. 37, 599-609 (2017).

9. Loftus, G. R. Eye fixations and recognition memory for pictures. Cogn. Psychol. 3, 525$551(1972)$.

10. Molitor, R. J., Ko, P. C., Hussey, E. P., \& Ally, B. A. Memory-related eye movements challenge behavioral measures of pattern completion and pattern separation. Hippocampus 24, 666-672 (2014). 
1 11. Olsen, R. K., Sebanayagam, V., Lee, Y., Moscovitch, M., Grady, C. L., Rosenbaum, R. S., \& Ryan, J. D. The relationship between eye movements and subsequent recognition: Evidence from individual differences and amnesia. Cortex 85, 182-193 (2016).

12. Brandt, S. A., \& Stark, L. W. Spontaneous eye movements during visual imagery reflect the content of the visual scene. J. Cogn. Neurosci. 9, 27-38 (1997).

13. Laeng, B., \& Teodorescu, D.-S. Eye scanpaths during visual imagery reenact those of perception of the same visual scene. Cogn. Sci. 26, 207-231 (2002).

14. Gbadamosi, J., \& Zangemeister, W. H. Visual imagery in hemianopic patients. J. Cogn. Neurosci. 13, 855-866 (2001).

15. Johansson, R., Holsanova, J., \& Holmqvist, K. Pictures and spoken descriptions elicit similar eye movements during mental imagery, both in light and in complete darkness. Cogn. Sci 30, 1053-1079 (2006).

16. Johansson, R., Holsanova, J., Dewhurst, R., \& Holmqvist, K. Eye movements during scene recollection have a functional role, but they are not reinstatements of those produced during encoding. J. Exp. Psychol. Hum. Percept. Perform. 38, 1289-1314 (2012).

17. Richardson, D. C., \& Spivey, M. J. Representation, space and Hollywood Squares: looking at things that aren't there anymore. Cognition 76, 269-295 (2000).

18. Ryan, J. D., Althoff, R. R., Whitlow, S., \& Cohen, N. J. Amnesia is a deficit in relational memory. Psychol. Sci. 11, 454-461 (2000).

19. Ferreira, F. Apel, A., \& Henderson, J. M. Taking a new look at looking at nothing. Trends Cogn. Sci. 12, 405-410 (2008).

20. Richardson, D. C., Altmann, G. T. M., Spivey, M. J., \& Hoover, M. A. Much ado about eye movements to nothing: a response to Ferreira et al.,: Taking a new look at looking at nothing, Trends Cogn. Sci. 13, 235-236 (2009). 
21. Wynn, J. S., Shen, K., \& Ryan, J. D. Eye Movements Actively Reinstate Spatiotemporal Mnemonic Content. Vision, 3, 21 (2019).

22. Hebb, D. O. Concerning imagery. Psychol. Rev. 75, 466-477 (1968).

23. Neisser, U. Cognitive psychology (Appleton-Century-Crofts, 1967).

24. Noton, D., \& Stark, L. Scanpaths in saccadic eye movements while viewing and recognizing patterns. Vis. Res. 11, 929-942 (1971).

25. Noton, D., \& Stark, L. W. Scanpaths in eye movements during perception. Science 171, 308-311 (1971).

26. Laeng, B., Bloem, I. M., D’Ascenzo, S., \& Tommasi, L. Scrutinizing visual images: the role of gaze in mental imagery and memory. Cognition 131, 263-283 (2014).

27. Wynn, J. S., Olsen, R. K., Binns, M. A., Buchsbaum, B. R., \& Ryan, J. D. Fixation reinstatement supports visuospatial memory in older adults. J. Exp. Psychol. Hum. Percept. Perform. 44, 1119-1127 (2018).

28. Bone, M. B., St-Laurent, M., Dang, C., McQuiggan, D. A., Ryan, J. D., \& Buchsbaum, B. R. Eye movement reinstatement and neural reactivation during mental imagery. Cereb. Cortex 29, 1075-1089 (2018).

29. Johansson, R., Bramao, I., Dewhurst, R., \& Johansson, M. Neurocognitive bases for the functional role of gaze direction during episodic memory retrieval. The $25^{\text {th }}$ Annual Meeting of the Cognitive Neuroscience Society, Boston, US, March 24-27 (2018).

30. Johansson, R., \& Johansson, M. Look here, eye movements play a functional role in memory retrieval. Psychol. Sci. 25, 236-242 (2014).

31. Johansson, R., \& Johansson, M. Gaze position regulates memory accessibility during competitive memory retrieval. Cognition 197, 104169 (2020).

32. Scholz, A., Mehlhorn, K., \& Krems, J. F. Listen up, eye movements play a role in verbal memory retrieval. Psychol. Res. 80, 149-158 (2016). 
33. Scholz, A., Klichowicz, A., \& Krems, J. F. Covert shifts of attention can account for the functional role of "eye movements to nothing". Mem. Cogn. 46, 230-243 (2018).

34. Van Ede, F., Chekroud, S. R., \& Nobre, A. C. Human gaze tracks attentional focusing in memorized visual space. Nat. Hum. Behav. 3, 462-470 (2019).

35. Dewhurst, R., Nyström, M., Jarodzka, H., Foulsham, T., Johansson, R., \& Holmqvist, K. It depends on how you look at it: Scanpath comparison in multiple dimensions with MultiMatch, a vector-based approach. Behav. Res. Methods 44, 1079-1100 (2012).

36. Pärnamets, P., Johansson, R., Gidlöf, K., \& Wallin, A. How information availability interacts with visual attention during judgment and decision tasks. J. Behav. Decis. Mak. 29, 218-231 (2016).

37. Jarodzka, H., Holmqvist, K., \& Nyström, M. A vector-based, multidimensional scanpath similarity measure. In Proceedings of the 2010 symposium on eye-tracking research \& applications, 211-218 (2010).

38. Anderson, N. C., Anderson, F., Kingstone, A., \& Bischof, W. F. A comparison of scanpath comparison methods. Behav. Res. Methods 47, 1377-1392 (2015).

39. Dewhurst, R., Foulsham, T., Jarodzka, H., Johansson, R., Holmqvist, K., \& Nyström, M. How task demands influence scanpath similarity in a sequential number-search task. Vis. Res. 149, 9-23 (2018).

40. Foulsham, T., Dewhurst, R., Nyström, M., Jarodzka, H., Johansson, R., Underwood, G., \& Holmqvist, K. Comparing scanpaths during scene encoding and recognition: A multidimensional approach. J. Eye Mov. Res. 5, 1-14 (2012).

41. Frame, M. E., Warren, R., \& Maresca, A. M. Scanpath comparisons for complex visual search in a naturalistic environment. Behav. Res. Methods 51, 1454-1470 (2019). 
42. Foulsham, T., \& Underwood, G. (2008). What can saliency models predict about eye movements? Spatial and sequential aspects of fixations during encoding and recognition. J. Vis. 8, 1-17 (2008).

43. Wynn, J. S., Ryan, J. D., \& Buchsbaum, B. R. Eye movements support behavioral pattern completion. Proc. Natl. Acad. Sci. 117, 6246-6254 (2020).

44. Foulsham, T., \& Kingstone, A. (2013). Fixation-dependent memory for natural scenes: An experimental test of scanpath theory. J. Exp. Psychol. Gen. 142, 41-56 (2013).

45. Bochynska, A., \& Laeng, B. Tracking down the path of memory: eye scanpaths facilitate retrieval of visuospatial information. Cogn. Process. 16, 159-163 (2015).

46. Kragel, J. E., \& Voss, J. L. Temporal context guides visual exploration during scene recognition. J. Exp. Psychol. Gen. Advance online publication (2020).

47. Eichenbaum, H., Yonelinas, A. P., \& Ranganath, C. The medial temporal lobe and recognition memory. Annu. Rev. Neurosci. 30, 123-152 (2007).

48. Danker, J. F., \& Anderson, J. R. The ghosts of brain states past: Remembering reactivates the brain regions engaged during encoding. Psychol. Bull. 136, 87-102 (2010).

49. Kent, C., \& Lamberts, K. The encoding-retrieval relationship: retrieval as mental simulation. Trends Cogn. Sci. 12, 92-98 (2008).

50. Tulving, E., \& Thomson, D. M. Encoding specificity and retrieval processes in episodic memory. Psychol. Rev. 80, 352-373 (1973).

51. Roediger, H. L., III, \& Guynn, M. J. Retrieval processes. Memory: Handbook of perception and cognition (eds. Bjork, E. L., \& Bjork, R. A.) 237-313 (Academic Press, 1996).

52. Bridge, D. J., Cohen, N. J., \& Voss, J. L. Distinct hippocampal versus frontoparietal network contributions to retrieval and memory-guided exploration. J. Cogn. Neurosci. 29, 1324-1338 (2017). 
1 53. Hannula, D. E., \& Ranganath, C. The eyes have it: hippocampal activity predicts expression of memory in eye movements. Neuron 63, 592-599 (2009).

54. Ryals, A. J., Wang, J. X., Polnaszek, K. L., \& Voss, J. L. Hippocampal contribution to implicit configuration memory expressed via eye movements during scene exploration. Hippocampus 25, 1028-1041 (2015).

55. Smith, C. N., \& Squire, L. R. Experience-dependent eye movements reflect hippocampus-dependent (aware) memory. J. Neurosci. 28, 12825-12833 (2008).

56. Champely, S. pwr: Basic functions for power analysis. R package version 1.1-3. 2015 (2016).

57. Peirce, J., Gray, J.R., Simpson, S., MacAskill, M., Höchenberger, R., Sogo, H., Kastman, research. Preprint at https://arxiv.org/abs/1505.03581 (2015).

60. Bates, D., Mächler, M., \& Bolker, B. S. Walker. Fitting Linear Mixed-Effects Models Using lme4. J. Stat. Softw. 67, 1-48 (2015). 


\section{Figures}

\section{A. Stimuli examples}

Scenes

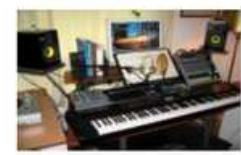

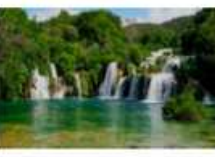
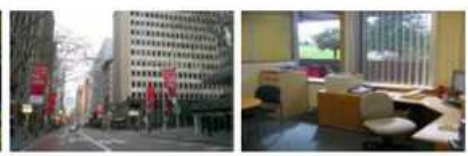

Object arrangements

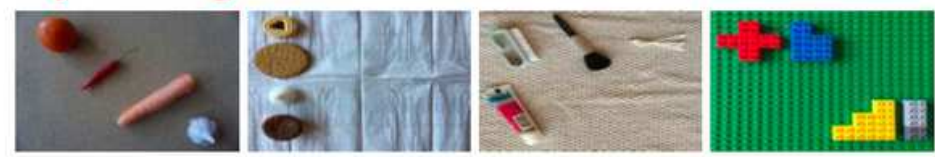

\section{B. Experimental design}

\section{Encoding}

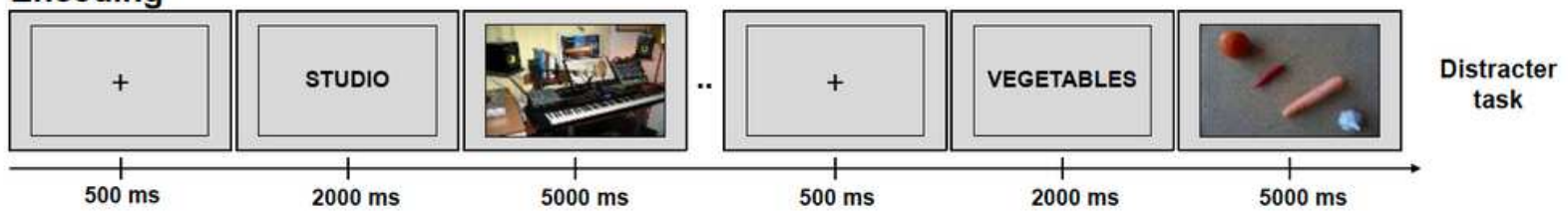

Recall

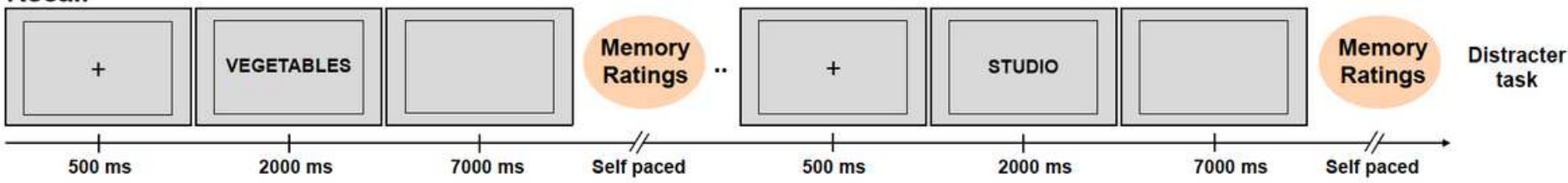

Surprise test: 4AFC Recognition test

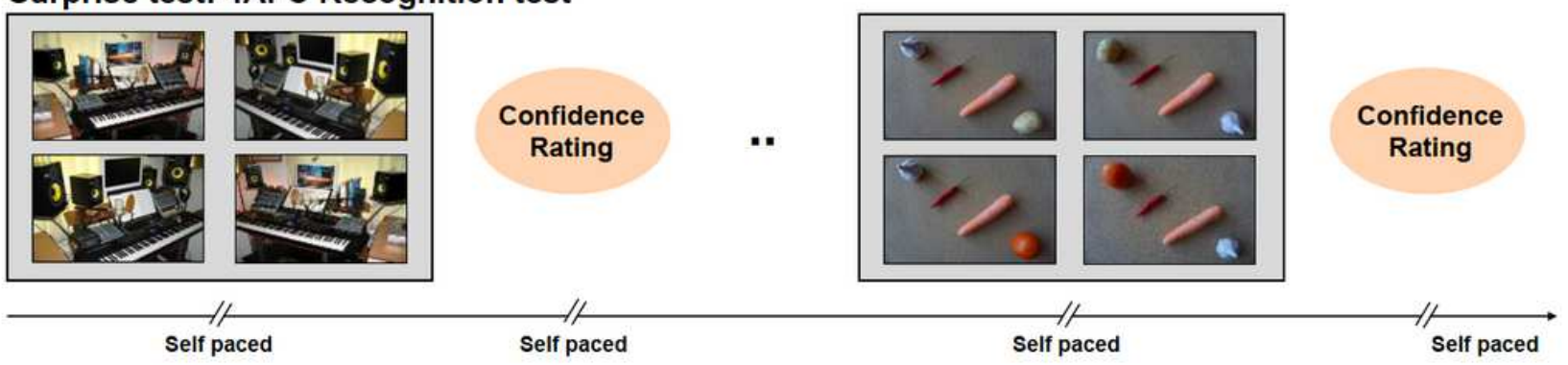

\section{Figure 1}

Encoding and retrieval of scenes and object arrangements. (A) Example of stimuli images (Scenes: studio, waterfalls, city street, office; Object arrangements: vegetables, cookies, bathroom things, lego). (B) Experimental design. In an initial encoding phase, participants encode 36 images of the two stimuli types (18 scenes, 18 object arrangements), preceded by associated descriptive labels. In a subsequent recall phase, the descriptive labels are presented as cues for memory retrieval, and participants recall each image while looking at a blank screen. After each recollection, they rate the quality (overall memory, vividness, spatial accuracy) of their recollection ( $0-100 \%)$. After a full round of encoding and recall, they then reach a surprise test, where each encoded and recalled image is presented in a four-alternative forced-choice (4AFC) recognition task that includes three similar distracters. Participants chose the specific image they believe they had encoded and recalled, and rate their confidence in this selection $(0-$ $100 \%)$. 
A

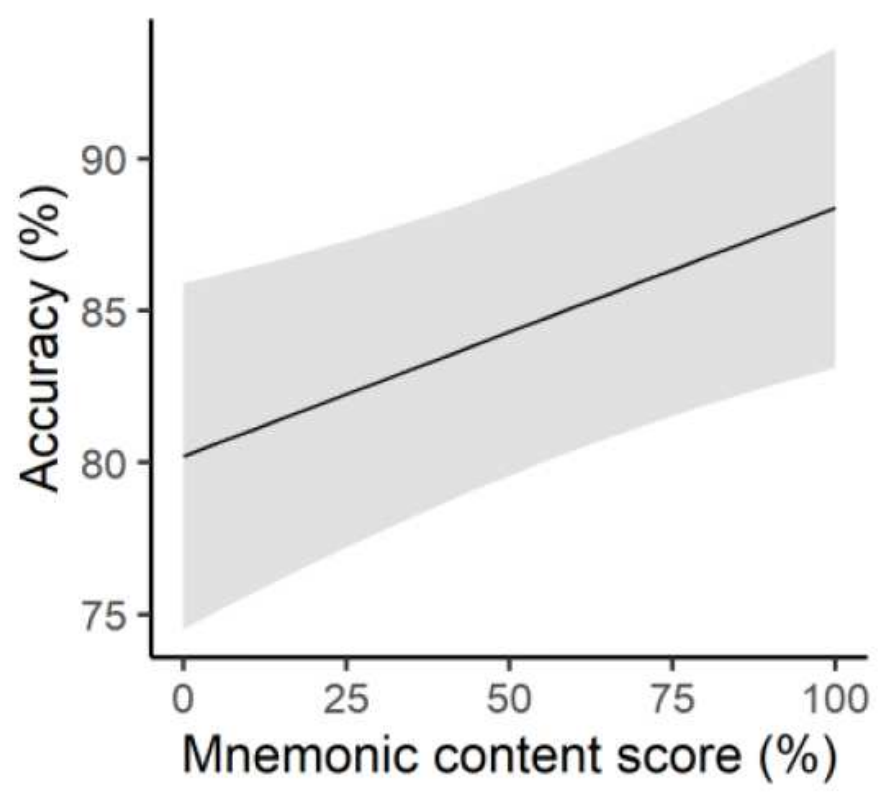

C

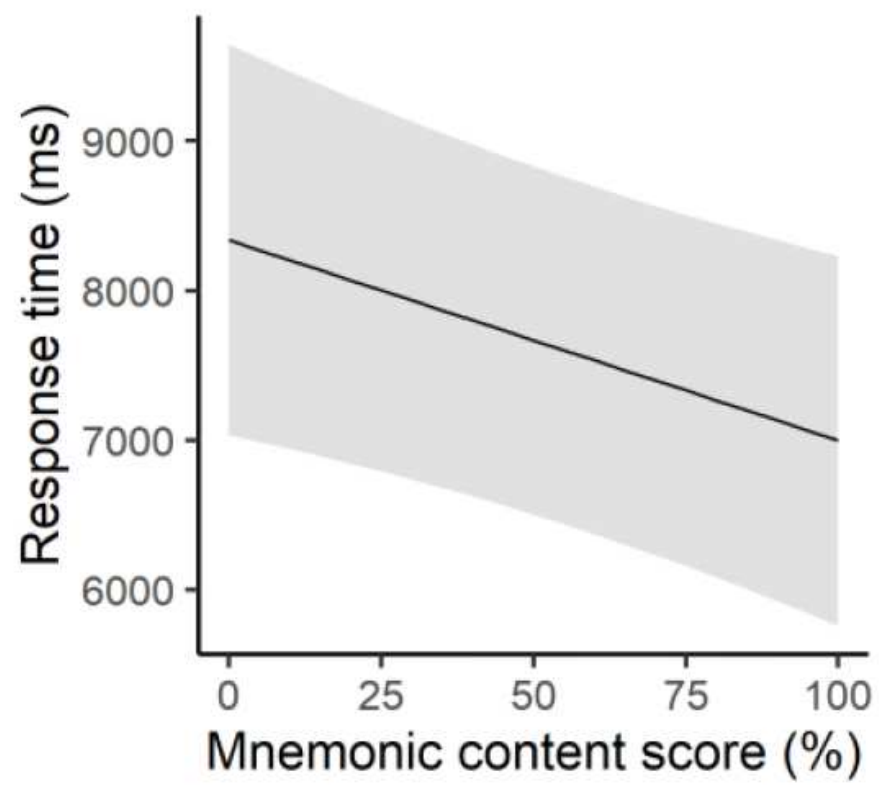

\section{B}

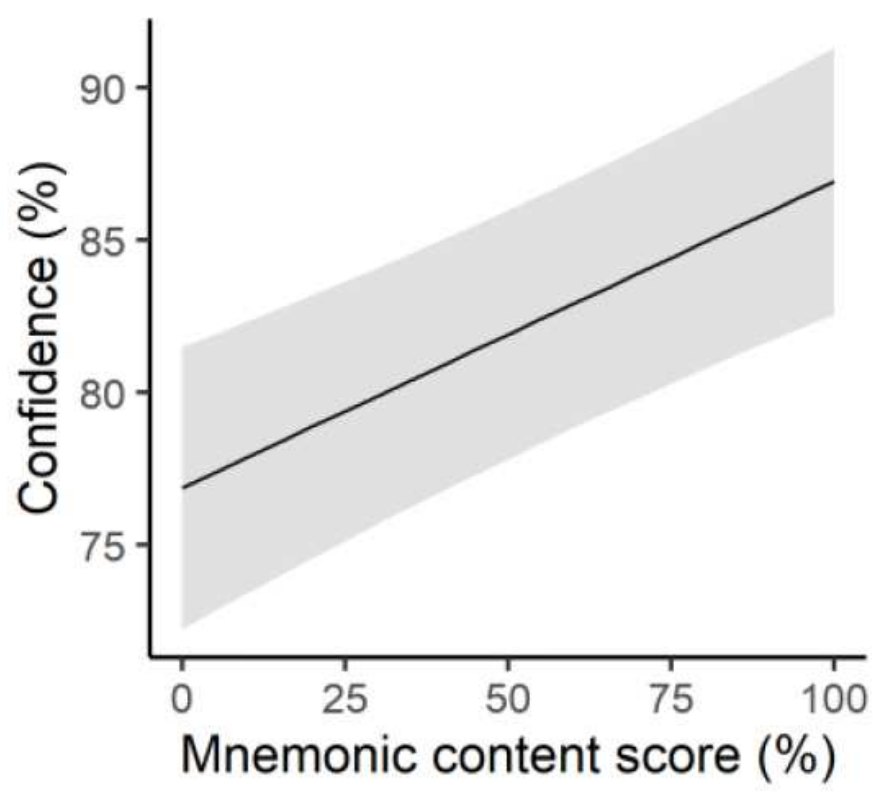

D

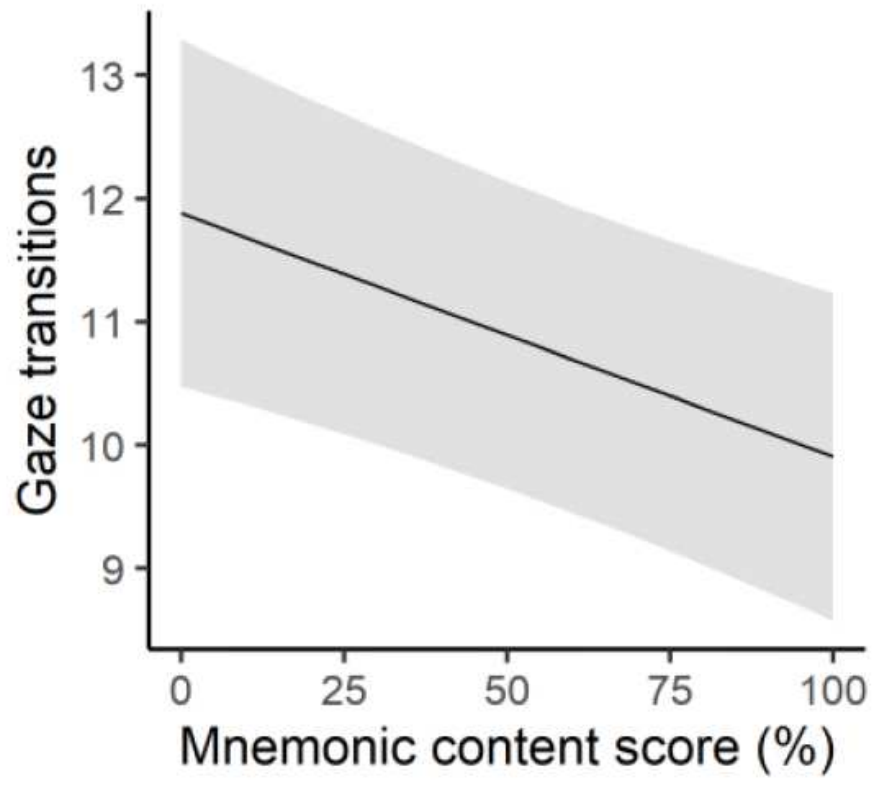

Figure 2

Memory quality during recall predicts subsequent recognition. How the mnemonic content score from the recall phase predicted (A) accuracy, (B) confidence, (C) response time, and (D) choice certainty in the corresponding 4AFC recognition task. Error bars and shaded areas denote $95 \%$ Confidence Intervals. 


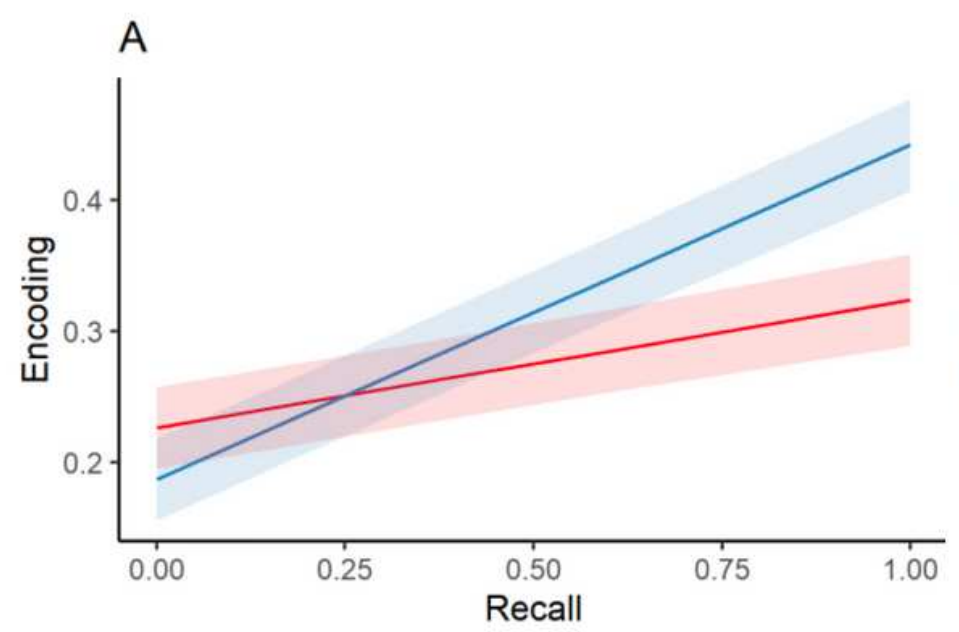

Scenes

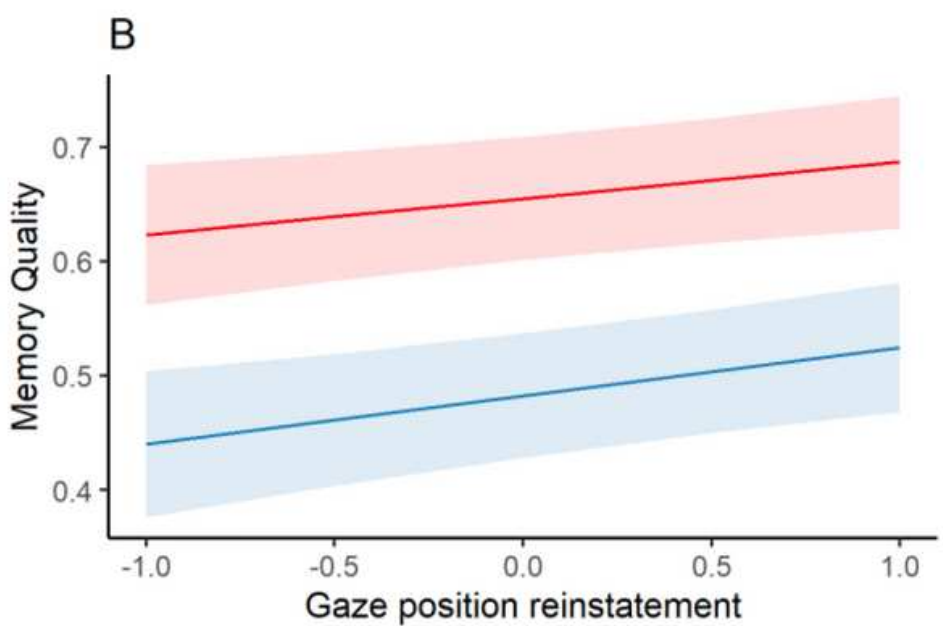

Object arrangements

\section{Figure 3}

Spatial gaze reinstatement. (A) Reinstatement of gaze positions over the two image types (scenes, objects). The $x$ - and $y$-axis corresponds to proportion gaze within the areas of interest on the presentation frame (see supplementary details on results). (B) The relationship between the degree of gaze position reinstatement and memory quality during recall. Shaded areas denote $95 \%$ Confidence Intervals.

\section{A. Scanpath Similarity analysis (MultiMatch)}

Scanpath 1
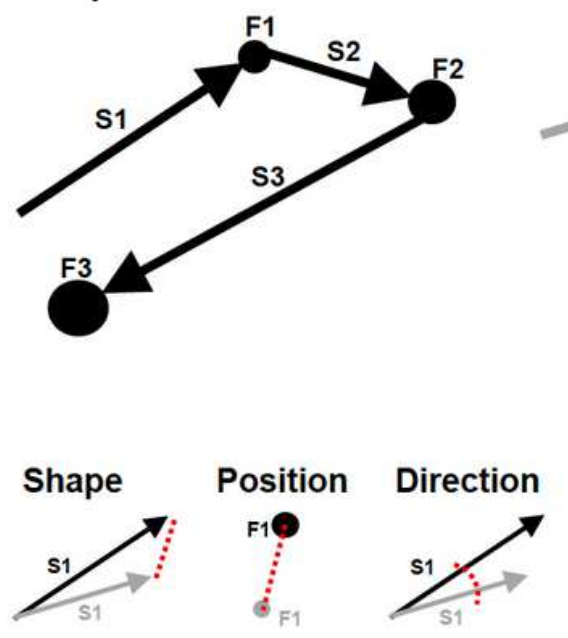
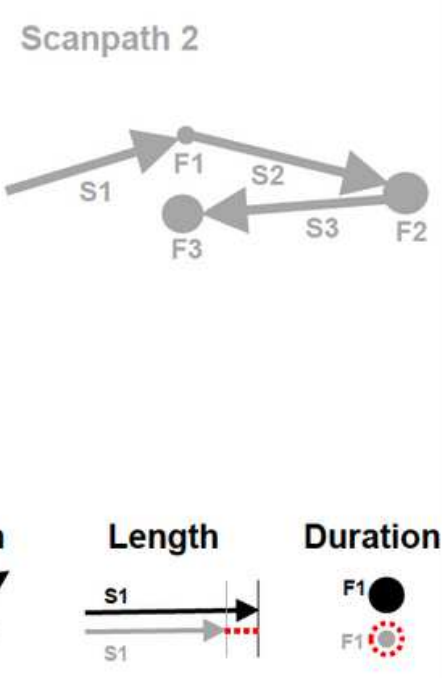

B. Examples of varying Encoding-Retrieval similiarity

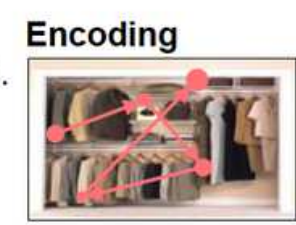

\section{Recall}

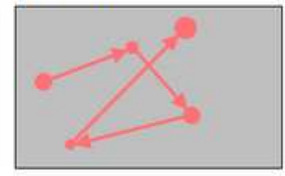

High Position

High Shape

High Direction

High Length

High Duration

ii.
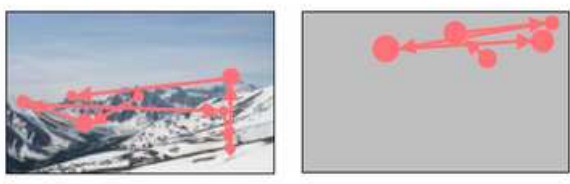

Low Position High Shape Low Direction Low Length Low Duration

iii.
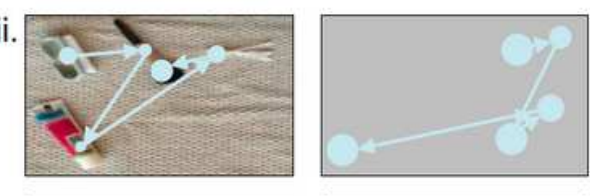

iv.

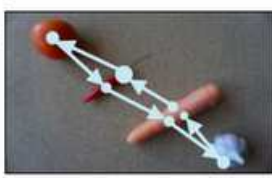

Low Position Low Shape High Direction Low Length

Low Duration

Low Position Low Shape Low Direction Low Length Low Duration

Figure 4

Scanpath similarity. (A) Overview of the MultiMatch scanpath similarity analysis. The top panel shows two relatively similar scanpaths under comparison, where fixations are represented as dots (F1-F3) and 
saccades as arrows between the fixations (S1-S3). Larger dots represent longer fixation duration. The bottom panel illustrates differences over the five dimensions of shape, position, direction, length and duration for the first pairs of aligned saccades (S1) and fixations (F1). The numeric difference between each dimension is illustrated with a red dotted line for each dimension separately. The Position dimension relies on spatial coordinates in absolute space and quantifies how similar aligned fixations are in respect to Euclidean distances. In contrast, the Shape, Direction and Length dimensions rely on differences in relative space. The Shape dimension quantifies how similar aligned saccadic vectors are in overall geometric shape, whereas the Direction dimension quantifies how similar aligned saccadic vectors are in geometric angle. The Length dimension quantifies how similar aligned saccades are in their absolute amplitude. The Duration dimension does not rely on any spatial coordinates and quantifies how similar aligned fixations are in their duration. (B) Examples of varying encoding-retrieval similarity: (i) complete encoding-retrieval overlap in respect to all five MM-dimensions; (ii) relatively high encodingretrieval similarity in Shape, but relatively low similarity in Position (due to dislocation in absolute space during recall), Direction (due to large differences in saccadic angles during recall), Length (due to overall shorter saccades during recall), and Duration (due to overall longer fixation durations during recall); (iii) high encoding-retrieval similarity in Direction, but low similarity in Shape (due to local differences in saccadic lengths during recall), Position (due to dislocation in absolute space during recall), Length (due to disproportional saccadic lengths during recall), and Duration (due to overall longer fixation durations during recall); (iv) low similarity over all five MM-dimensions.

A

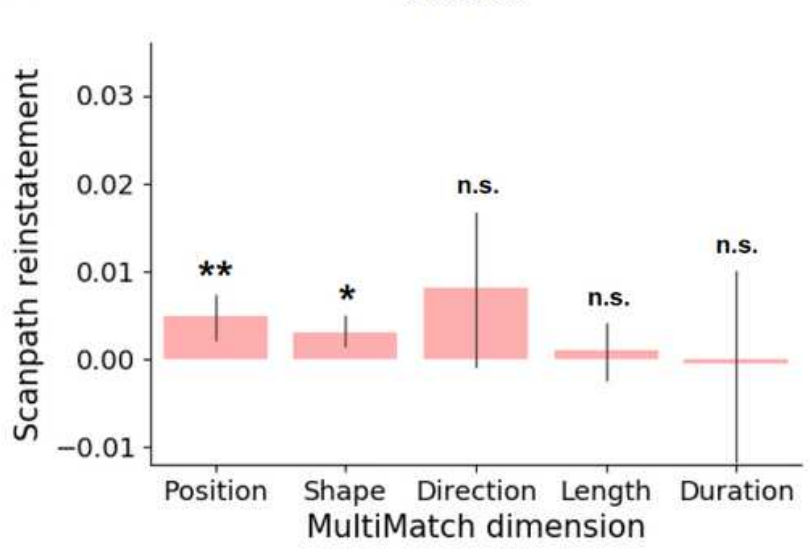

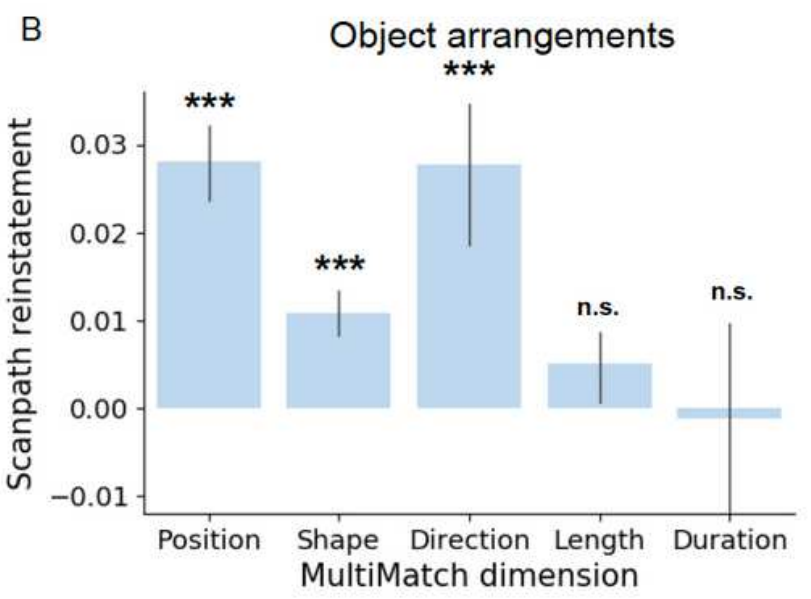

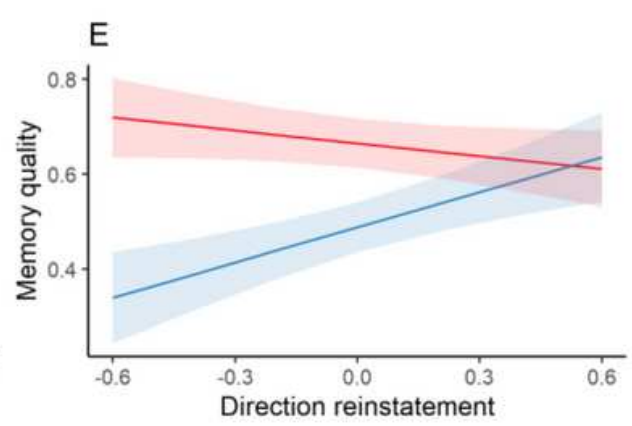

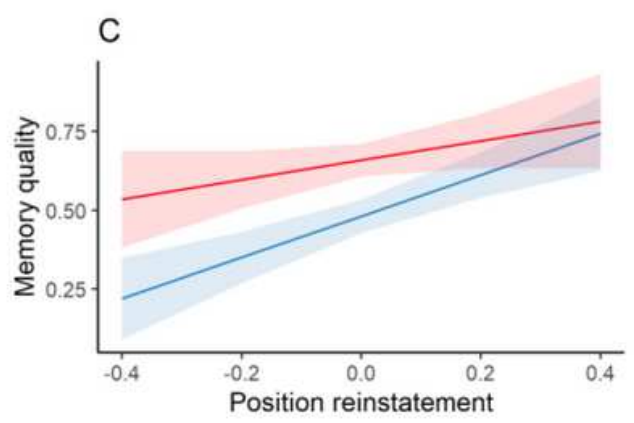

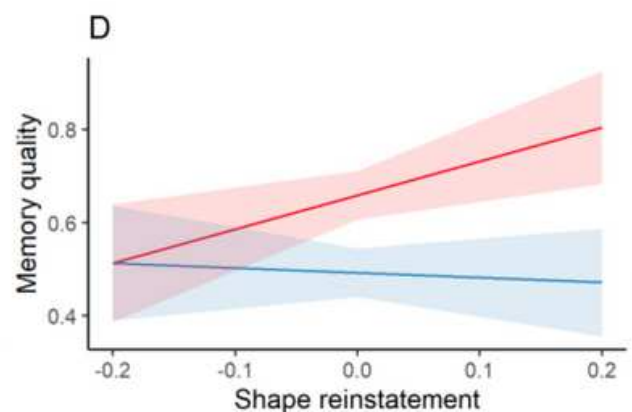

Scenes
Object arrangements 


\section{Figure 5}

Encoding-retrieval similarity over different scanpath dimensions. Scanpath reinstatement over the five dimensions for the (A) scenes and (B) object arrangements. The measure of scanpath reinstatement represents the difference between encoding-retrieval similarity and baseline similarity (a value greater than zero indicates scanpath reinstatement). (C) The relationship between encoding-retrieval similarity in position and memory quality. (D) The relationship between encoding-retrieval similarity in shape and memory quality. $(E)$ The relationship between encoding-retrieval similarity in direction and memory quality. Error bars and shaded areas denote $95 \%$ Confidence Intervals, $* p<.05,{ }^{\star *} p<.01, * \star \star p<.001$.

\section{Supplementary Files}

This is a list of supplementary files associated with this preprint. Click to download.

- NatBehavSupplMatJohanssonetal2010VRSCanpaths20210222.docx 